\title{
Successful Endovascular Treatment of Post-Traumatic Carotid -Cavernous Fistula of Flow-Diverter Stents
}

\author{
AA Sufianov ${ }^{1,2}, S M$ Karasev $^{1}, R R$ Khafizov $^{1 *}$ and RA Sufianov ${ }^{2}$ \\ ${ }^{1}$ Federal Center for Neurosurgery of the Ministry of Health of Russia, Tyumen, Russia \\ ${ }^{2}$ First Moscow State Medical University named after IM Sechenov Ministry of Health of Russia, Moscow, Russia \\ *Corresponding author: RR Khafizov, Interventional Radiologist, Federal Center for Neurosurgery of the Ministry of \\ Health of Russia, Tyumen, Russia, Tel: +79174360060
}

\begin{abstract}
Carotid - cavernous fistulas (CCF) are spontaneous or acquired communications between the internal carotid artery (ICA) and the cavernous sinus, which can be classified as direct or indirect. Direct fistulas between the internal carotid artery and the cavernous sinus can occur due to trauma, ruptures of intracavernous carotid artery aneurysms, collagen deficiency syndromes, stratification of arteries, fibromuscular dysplasia, and direct surgical trauma. Symptoms caused by CCF are associated with their size, duration, location, direction of the venous outflow, as well as the presence of arterial and venous collaterals. The goal of treating direct CCF is to close the defect between the ICA and the cavernous sinus while maintaining the patency of the ICA. The choice of treatment is carried out in accordance with the type, exact anatomy of the fistula, the size of the arterial defect and the preferences of the operator. The issue of choosing a treatment for CCF remains relevant. The article presents a clinical case of successful endovascular treatment of post-traumatic carotid-cavernous fistula type $A$. The reconstruction of the carotid-cavernous fistula with flow-deflecting stents according to the "telescope" technique was performed.
\end{abstract}

\section{Keywords}

Carotid-cavernous fistula, Embolization by coils, Flow-diverter stents, "Telescope" technique stenting

\section{Introduction}

Carotid - cavernous fistulas (CCF) are abnormal connections between the internal carotid artery and the cavernous sinus. Some circuits classified CCF according to etiology (traumatic or spontaneous), hemodynamic features (high or low speed), or angiographic arterial architecture (direct or indirect). Angiographic classification defines the angioarchitecture of the lesion on which the endovascular strategy of treatment can be planned. According to angiographic data, Barrow, et al. [1] provided a detailed anatomical classification that classifies CCF into four different types. Type A fistula is a direct connection between the internal carotid artery (ICA) and the cavernous sinus, usually associated with a high flow rate. Indirect fistulas (types B, C, and D) are dural arteriovenous fistulas that are fed by the ICA meningeal arteries, the external carotid artery (ECA), or both. Type $B$ fistulas have dural branches of the ICA into the cavernous sinus, which are relatively rare. Type $C$ fistulas are represented exclusively by dural branches of the HCA. The most common form of indirect CCF is a type D fistula, which has dural ICA and ECA branches to the cavernous sinus. Tomsick, et al. classifies type D CCF into type D1 or D2 depending on the presence of unilateral or bilateral arterial supply [2].

Traumatic destruction of the vessel wall is the most common etiological factor for direct CCF $[3,4]$.

Recent advances in endovascular technology have led to a number of different treatment options for CCF. Although the clinical manifestations of direct and indirect fistulas may overlap, their natural history and method of endovascular treatment are often significantly different. The choice of treatment is carried out in accordance with the type, exact anatomy of the fistula, the size of the arterial defect and the

Citation: Sufianov AA, Karasev SM, Khafizov RR, Sufianov RA (2019) Successful Endovascular Treatment of Post-Traumatic Carotid -Cavernous Fistula of Flow-Diverter Stents. Neurosurg Cases Rev 2:029. doi.org/10.23937/2643-4474/1710029

Accepted: November 02, 2019; Published: November 04, 2019

Copyright: (c) 2019 Sufianov AA, et al. This is an open-access article distributed under the terms of the Creative Commons Attribution License, which permits unrestricted use, distribution, and reproduction in any medium, provided the original author and source are credited. 


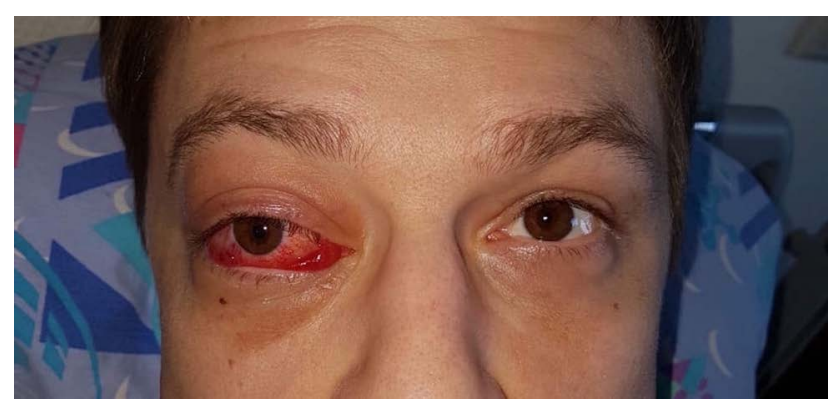

Figure 1: Patient's appearance: Exophthalmos, chemosis, "bloody tears", injection of sclera.

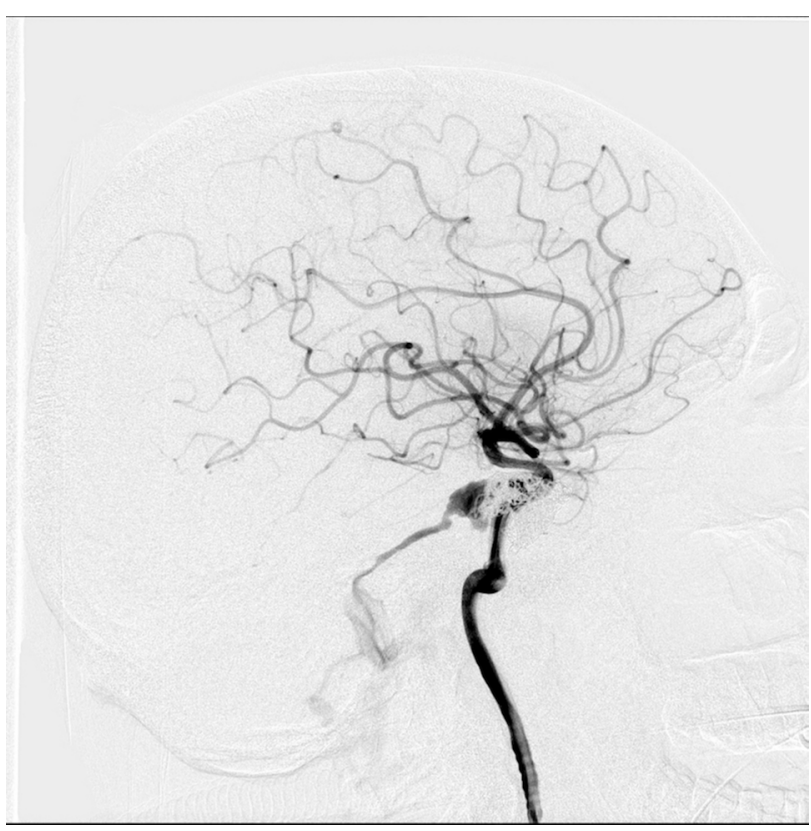

Figure 2: Angiography of the internal carotid arteries (ICA), lateral projection: Carotid-cavernous fistula of the right ICA with discharge into the upper stony sinus. Condition after previous embolization.

A)

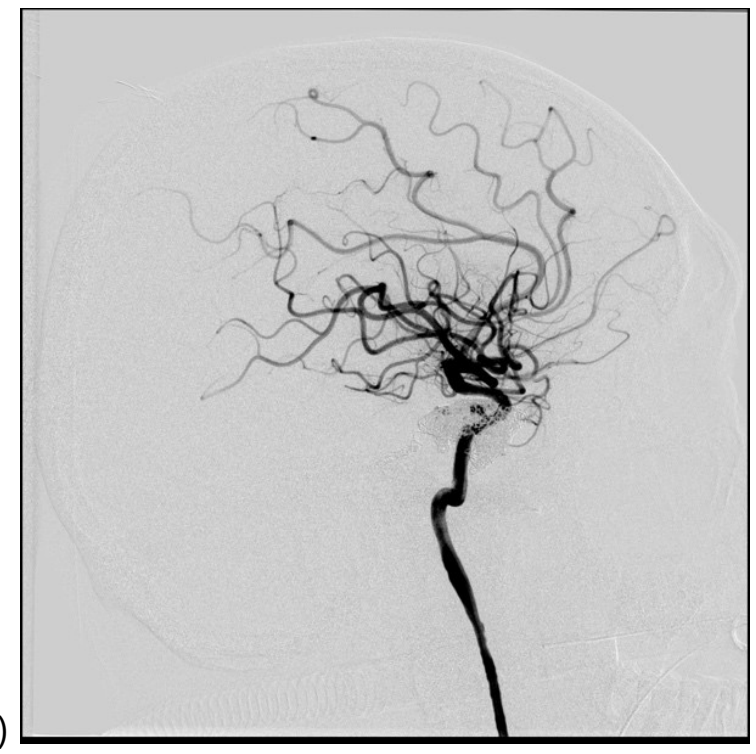

B)



Figure 4: Cerebral angiography, lateral projection

A) Arterial phase, B) venous phase. Fully restored blood flow in the right ICA 
planned surgical treatment with complaints of visual impairment, swelling of the right eye, protrusion. Anamnesis: About two years ago patient received a head injury. Previously examined and treated in another clinic. A carotid-cavernous fistula was revealed, embolization with coils was performed. In the postoperative period, no improvement was observed. The phenomena of pulsating exophthalmos, conjunctival chemosis, ischemic optic neuropathy of the right eye persisted (Figure 1).

Diagnosis: Carotid-cavernous fistula type A on the right. Condition after endovascular occlusion of the cavity of the carotid-cavernous fistula on the right with coils. Right exophthalmos. Ischemic optoneuropathy of the right eye.

In order to determine further treatment tactics, the patient underwent selective cerebral angiography: According to the selective cerebral angiography data, a type A carotid-cavernous fistula was revealed (Figure 2).

Given the nature of the lesion, it was decided to implant a flow-diverter stent FRED ${ }^{\circledR}$ (MicroVention).

\section{Technique}

Under general anesthesia, a puncture of the right femoral artery was performed. An angiographic catheter $5 \mathrm{~F}$ on a guidewire $0.035^{\prime \prime}$ was inserted through the inserted introducer 6F. Catheterized right ICA. Contrast the right ICA, the pool of the right ICA. Identified carotid-cavernous fistula type $A$, distal blood flow preserved. Considering the presence of a preserved distal channel and a defect in the vascular wall of the ICA of large sizes, we decided to implant two stent-diverter stents using the "telescope" technique.

Angiographic catheter is replaced by a guiding catheter. A microcatheter is installed in the lumen of the right ICA and the FRED diverter stents are precisely positioned and alternately implanted.

On control angiography, the complete restoration of the ICA lumen with no discharge into the cavernous sinus is determined, the main and distal vessels

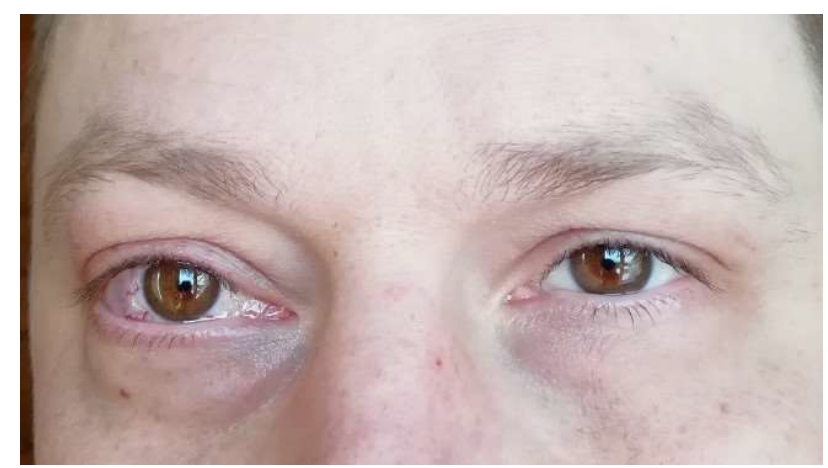

Figure 5: The appearance of the patient 3 months after surgery. Reducing edema of the right eye, the absence of exophthalmos, conjunctival chemosis. are passable (Figures 3 and Figure 4). Microcatheter removed without technical difficulties. Endovascular instrumentation removed. Hemostasis, bleeding. A pressure bandage was applied to the puncture site.

The early postoperative period was uneventful. The dressing was removed after 24 hours. The pulse on the femoral artery is preserved. Neurological status of the patient without features. Discharged from the hospital in satisfactory condition on the $3^{\text {rd }}$ day after the intervention.

In the long-term period, three months after the intervention, a significant decrease in exophthalmos, conjunctival chemosis, and scleral injections is determined. A satisfactory aesthetic and clinical result of the operation is noted. The patient notes an improvement in vision (Figure 5).

\section{Discussion}

Diagnostic and treatment of CCF has improved significantly in recent years. The widespread availability of non-invasive imaging methods, combined with improvements in catheter angiography, allows in most cases to be quickly and accurately diagnosed, and new endovascular methods can successfully treat most patients with these lesions with virtually no complications or death, and with the permission of most clinical manifestations [6].

In our case, we chose this strategy for the following reasons: Given the early ineffective coils embolization, the possible development of the "mass - effect", venous insufficiency after complete occlusion with the coils. We used the "telescopic" technique of implantation of two flow - diverter stents in order to completely reconstruct the cavernous section of the ICA.

\section{Conclusion}

Thus, this clinical case showed the efficacy and safety of the use of flow-diverter stents in this pathology. In our opinion, the use of the "telescopic" technique of stent implantation in this situation was the most justified, in view of the extensive defect in the vascular wall.

\section{References}

1. Barrow DL, Spector RH, Braun IF, Landman JA, Tindall SC, et al. (1985) Classication and treatment of spontaneous carotid-cavernous sinus stulas. J Neurosurg 62: 248-256.

2. Ernst RJ, Tomsick TA (1997) Classification and angiography of carotid cavernous fistulas. In: Tomsick TA, Carotid cavernous sinus fistula. Digital Education Publishing, Cincinnati, 13-22.

3. Tjoumakaris SI, Jabbour PM, Rosenwasser RH (2009) Neuro-endovascular management of carotid cavernous fistulae. Neurosurg Clin N Am 20: 447-452.

4. Connors JJ, Wojak JC (1999) Interventional neuroradiolo- 
gy: Strategies and practical techniques. WB Saunders Co, Philadelphia, 215-226.

5. Gemmete JJ, Ansari SA, Gandhi DM (2009) Endovascular techniques for treatment of carotid-cavernous fistula. $\mathrm{J}$
Neuroophthalmol 29: 62-71.

6. Miller NR (2007) Diagnosis and management of dural carotid-cavernous sinus fistulas. Neurosurg Focus 23: 12-13. 